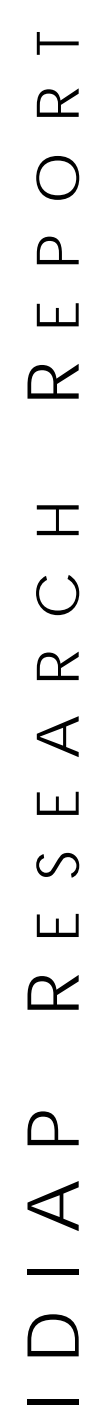

\section{A Multi-sample Multi-source MODEL FOR BIOMETRIC Authentication}

\author{
Norman Poh Hoon Thian ${ }^{a b}$ Samy Bengio ${ }^{a}$ \\ Jerzy Korczak ${ }^{\mathrm{b}}$ \\ IDIAP-RR 02-14 \\ Samy Bengio a
}

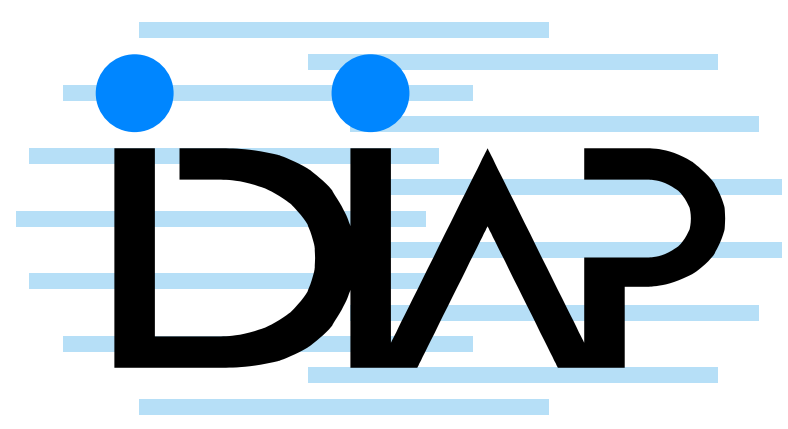

\begin{abstract}
APRIL 2002
PUBLISHED IN

2002 IEEE International Workshop on Neural Networks for Signal Processing (NNSP 2002), Martigny, Switzerland, pages 375-384,

September 2002.
\end{abstract}

Dalle Molle Institute for Perceptual Artificial Intelligence • P.O.Box 592 • Martigny $\bullet$ Valais $\bullet$ Switzerland phone $+41-27-721 \quad 77 \quad 11$ $\begin{array}{llll}\text { fax } \quad+41-27-721 & 77 & 12\end{array}$ e-mail secretariat@idiap.ch internet http://www.idiap.ch 

IDIAP Research Report 02-14

\title{
A Multi-Sample Multi-Source Model for Biometric Authentication
}

\author{
Norman Poh Hoon Thian \\ Samy Bengio \\ Jerzy Korczak
}

APRIL 2002

PUBLISHED IN

2002 IEEE International Workshop on Neural Networks for Signal Processing (NNSP 2002), Martigny, Switzerland, pages 375-384, September 2002.

\begin{abstract}
In this study, two techniques that can improve the authentication process are examined: (i) multiple samples and (ii) multiple biometric sources. We propose the fusion of multiple samples obtained from multiple biometric sources at the score level. By using the average operator, both the theoretical and empirical results show that integrating as many samples and as many biometric sources as possible can improve the overall reliability of the system. This strategy is called multi-sample multi-source approach. This strategy was tested on a real-life database using neural networks trained in one-versus-all configuration and evaluated on a validation set.
\end{abstract}




\section{Introduction}

Biometric authentication is the problem of verifying an identity claim using a person's behavioural and physiological characteristics. Biometric authentication is becoming an important alternative to traditional authentication methods such as keys ("something one has", i.e., by possession) or PIN numbers ("something one knows", i.e., by knowledge) because it is essentially "who one is", i.e., by biometric information. Therefore, it is not susceptible to misplacement, forgetfulness or reproduction. Examples of biometric sources are fingerprint, face, voice, hand-geometry and retina scans.

However, to date, biometric-based security systems (devices, algorithms, architectures) still have room for improvement, particularly in their accuracy, tolerance to various noisy environments and scalability as the number of individuals increases. The focus of this study is on minimising the noise by using multiple biometric sources and multiple samples.

Biometric data is often noisy because of deformable templates, corruption by environmental noise, variability over time and occlusion by the user's accessories. The higher the noise, the less reliable the biometric system becomes.

Advancements in biometrics show two emerging solutions: combining several biometric sources [6, $2,5]$ and combining several samples of a single biometric modality [3]. Combining several biometric sources can further be divided into a loosely coupled solution and a tightly coupled solution. A loosely coupled solution assumes very little or no interaction among the inputs. It integrates biometric data output of a relatively autonomous agent. An example of a loosely coupled system is the integration of audio and visual biometric data in an asynchronous manner. On the other hand, a tightly coupled solution assumes a strong interaction among the input measurements. It integrates biometric data at the sensor or representation level. An example of a tightly coupled system is the integration of audio and visual biometric data in a synchronous manner. In our opinion, combining several samples of a single biometric source can be considered a very tightly coupled solution because taking several life-scans of the same source of biometric data implies that the samples must be strongly correlated. Another category of solutions is to combine a biometric system with a non-biometric system.

Combining several biometric sources offers the advantage of relaxing the assumption of universality (the fact that each user should possess the biometric information), collectability (the extent to which the biometric information is measurable and adequately represented for the matching purpose), acceptability (the fact that each user agrees to have his/her biometric information scanned) and integrity (the degree of trustworthiness of the biometric system) of a target population in a given application.

Several studies have shown that a multi-source (modality) biometric system can improve the incompleteness of any single-model biometric system $[6,5]$. In particular, Hong et al. have proven both theoretically and empirically that integrating multiple biometric models at score level and decision level can improve the overall system accuracy [2]. Kittler et al. have shown that combining several samples of a single biometric source can also improve the accuracy of the overall system [3].

The purpose of this paper is to examine how the combined error could be reduced by using two separate approaches: multi-sample and multi-source. Section 2 gives an overview of a generic biometric framework and proposes a theoretical model to justify these two approaches. Section 3 shows some empirical results and is followed by our conclusions.

\section{Towards a multi-sample multi-source biometric solution}

\subsection{A generic biometric integration model}

In this section, a biometric-independent framework (see Figure 1) is proposed. Based on this framework, the process involving sensor, extractors, classifiers and supervisors is shown to exhibit a serial chain process. By using multiple samples, it will be shown that several such chain processes are "created" and that noise can be reduced. By using the concept of the "committee of classifiers" [1], it will be justified that by combining several biometric sources via averaging, more of such parallel process are created and that the system error will be reduced by averaging the output. In a system 
using multiple biometric samples and of different modalities, we will show that combining the score by averaging can reduce the misclassfication error further.

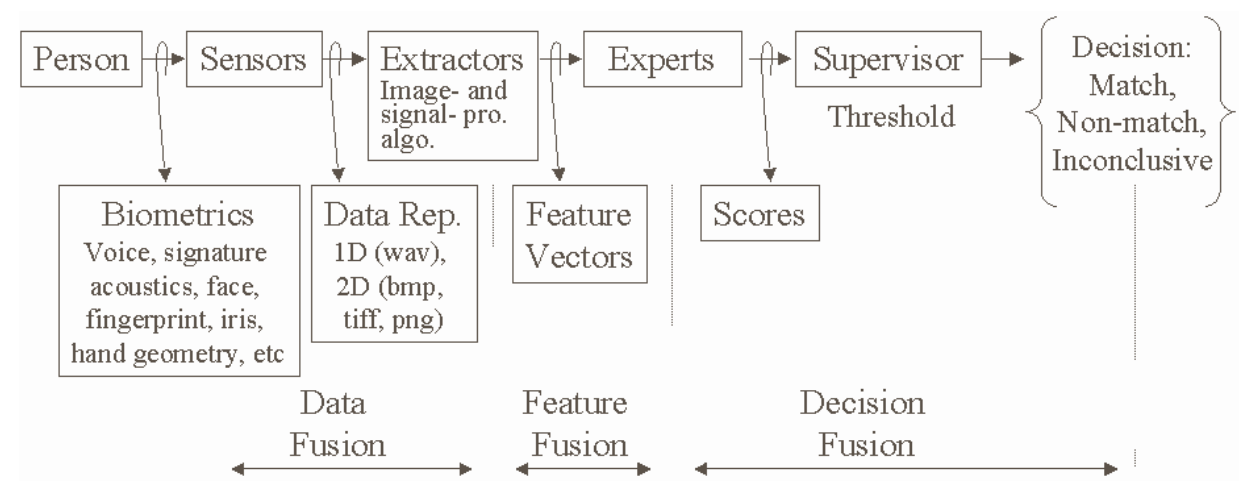

Figure 1: A generic biometric taxonomy and fusion scheme

In a biometric-independent framework (see Figure 1), a user's biometric data is captured using sensors. Examples of sensors are Charged Couple Device (CCD) cameras, Infrared-Red (IR) cameras, fingerprint scanners and microphones. Each sensors has its own standard data representation. A set of operations, often founded on signal- and image-processing algorithms, constitute the building blocks of extractors. Extractors have two functions: to detect and to extract user-discriminant information. Each extractor produces its own type of vectors or feature vectors, also called templates in a more generic setting. Experts or classifiers are used to recognise these produced vectors. Classifiers are a set of pattern-matching algorithms, which may be learning-based (e.g. Multi-Layer Perceptron, Support Vector Machine, etc) or template-based (dynamic time wrapping, Euclidean distance, normalised correlation, etc). Classifiers map a vector belonging to an associated identity. They do so with a certain degree of confidence commonly called a score or a confidence measure. It could be a scalar value or a vector when more information is supplied. A score could be interpreted as the estimated a posteriori probability that a given feature belongs to the claimed class label. When there are several classifiers, a supervisor merges different scores to obtain the final decision. If the final decision is a match, then the system accepts the identity claim. If the decision is a non-match, then the system rejects the identity claim. Finally, if the decision is inconclusive, a fallback procedure should be activated.

\subsection{Probability of failure of a single-modality biometric system}

In general, the core matching module of biometric system can be regarded as a function $f$ that receives a vector feature $\mathbf{x}$ and outputs a score $y: y=f(\mathbf{x})$. The function $f$ could be linear (e.g. Fisher discriminant analysis, Support Vector Machine with linear kernel) or non-linear (Multi-layer Perceptron, Support Vector Machines with non-linear kernel). $y$ represents a measurement. It could be a score $\in[-1,+1]$, a confidence score (a posteriori probability) $\in[0,1]$ or a distance metric $\in R^{+}$.

In the following section, $y$ will be a confidence score showing a posteriori probability (e.g. an MLP having a single output neuron using a sigmoid activation function). Let $p\left(w_{1} \mid y\right)$ be the probability distribution function $(p d f)$ of client confidence scores and $p\left(w_{2} \mid y\right)$ be the $p d f$ of impostor confidence scores.

The probability that a system commits error given a threshold $s$, which we denote as $E(s)$, can be calculated using:

$$
E(s)=\int P(\text { false rejection })+P(\text { false acceptance }) d y
$$




$$
=1-\int_{-\infty}^{s} p\left(w_{1} \mid y\right) d y+\int_{s}^{+\infty} p\left(w_{2} \mid y\right) d y .
$$

One seeks to minimise $E(s)$ such that $E_{\min }=\min _{s} E(s)$. The optimum threshold $s$ is at the point called Equal Error and $E_{\min }$ is called Equal Error Rate (EER). The assumption here is that the false acceptance and false rejection errors are considered equal. One can also define a cost function that give different weights to the false acceptance and the false rejection error.

It is obvious that if the two distributions completely overlap each other, $\mathrm{E}=1$ and if they do not overlap at all, $\mathrm{E}=0$. Note that in biometric applications, there are three categories of scores: genuine, "inter-template" (other clients) and impostors (also called "background database" [7]). There are also informed and uninformed impostors. In real-life hacking, impostors are informed, i.e., they possess a certain amount of information about the identity to be faked. Among these three major categories of scores stated earlier, the genuine user scores are often the smallest data set. This study considers only genuine users and impostors. From now onwards, impostors are taken as a union of "inter-template" and "background database".

\subsection{Classification of biometric system models}

Having studied the nature of chain process of biometric system and the error involved in such a system, one is led to study available types of biometric system models. In our opinion, biometric systems can be classified according to the number of samples per access and the number of biometric sources. The term "source" is used here to signify a particular class of biometric modality such as face, voice and so on. This is to distinguish it from the term "model" to be introduced later to signify different architecture. A "sample" is therefore a life-scan or shot of a biometric source. Using these two definitions, we propose four biometric "models". Figure 2(i) shows the typical serial process consisting of sensor, extractor and classifier. It is called a single-sample single-source (SSSS) biometric model. Figures 2(ii)-(iv) show a multi-sample single-source (MSSS), a single-sample multi-source (SSMS) and a multi-sample multi-source (MSMS) biometric model respectively. With this categorisation, Kittler et al.'s work [3] falls into MSSS model because several face samples are used during authentication. Hong et al.'s work [2] falls into SSMS model because they used a face sample and a fingerprint sample during authentication. Ross et al.'s work [6] that used face, fingerprint and hand geometry also falls into SSMS model.
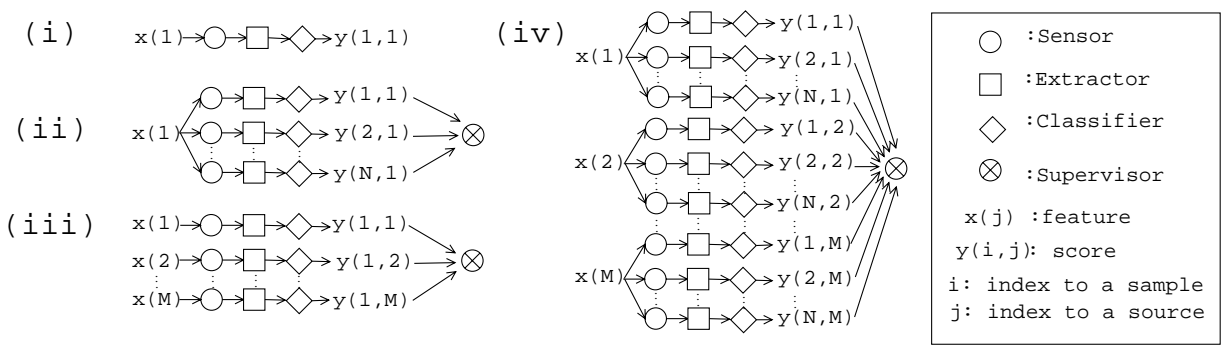

Figure 2: (i) Single-sample single-source, (ii) multi-sample single-source, (iii) single-sample multisource and (iv) multi-sample multi-source biometric model. $\mathrm{x}(\mathrm{j})$ and $\mathrm{y}(\mathrm{i}, \mathrm{j})$ are equivalent to $\mathbf{x}_{j}$ and $y_{i, j}$ in text respectively.

In Figure $2, \mathbf{x}_{j}$ is the $j$-th biometric source (or modality) and $j=1, \ldots, M . y_{i, j}$ is the score obtained from $i$-th sample of the $j$-th biometric source and $i=1, \ldots, N$. A MSSS model differs from a SSSS model in that a MSSS model uses several samples (therefore several serial processes) originating from the same biometric source. Note that a SSSS model does not require any supervisor. On the other hand, a SSMS model differs from a SSSS model in that a SSMS model uses several 
independent biometric sources. A MSSS model is more tightly coupled than a SSMS model. This means that if a biometric source is corrupted by the same noise (for instance, a cut on finger, a soar throat, a sun-burnt face), a MSSS model will probably fail to verify the identity of the person. On the other hand, multi-source biometric models (SSMS and MSMS) will probably be more robust against this kind of noise because their sources are not corrupted by the same noise.

\subsection{Multi-sample single-source approach}

Kittler et al. have shown that a MSSS model can be an effective way to boost a biometric system [3]. In their experiment setting with face images, several face samples are used. The optimal final decision score is found by averaging classifier scores. This can be justified by modelling a score corrupted by noise.

Let $y_{i}$ be the "observed" measure (i.e. a score, $y_{i}=f\left(\mathbf{x}^{i}\right)$ of a given sample $i$ out of a total of $N$ samples. There is a "true" measure $\hat{y}$ and it is corrupted by the noise $\eta_{i}$. This noise is assumed to be drawn from a random zero-mean additive distribution. The observed measure $y_{i}$ can be written as:

$$
y_{i}=\hat{y}+\eta_{i} .
$$

The mean of $y_{i}$, denoted as $\bar{y}$ is:

$$
\bar{y}=\frac{1}{N} \sum_{i=1}^{N} y_{i} .
$$

With enough samples, the expected value of $y_{i}$, denoted as $E\left\{y_{i}\right\}$, which is calculated as the mean of $y_{i}$ (Equation 4), approximates the "true" measure:

$$
E\left\{y_{i}\right\}=\hat{y} .
$$

The expected value of random noise $\eta_{i}$, i.e., $E\left(\eta_{i}\right)$ is always zero. The variance of the observed $y$ can be written as:

$$
\sigma_{y}^{2}=\frac{1}{N} \sigma_{\eta}^{2} .
$$

Therefore, it can be concluded that when two or more scores of a single modality biometric are averaged, noise that occurs due to classification can be reduced.

\subsection{Single-sample multi-source approach}

In this section, we would like to show that the error of the joint system is lower than the error of each of the sub-components when they operate independently. This can be represented as: $\forall_{j}\left(E_{d} \leq E_{j}\right)$, where $E_{j}$ is the error of one of $M$ sub-components $j$ and $E_{d}$ is the error of the joint system. Note that the index $j$ is used to signify a biometric source so as to distinguish it from the index $i$ that was used to signify one of $N$ samples taken from a given biometric modality.

It is desirable that the following relationship holds:

$$
E_{d}=\prod_{j=1}^{M} E_{j} .
$$

Equation 7 can be interpreted as follows: in a system with $M$ biometric sources, the whole system will always select the best sub-component. In other words, this is the optimal decision, called Oracle in [4]. Such a supervisor (one that merges the scores, as defined earlier) is the best result that one can get out of scores combination. From Equation 7, it is obvious that $\forall_{j}\left(E_{d} \leq E_{j}\right)$. Using normal and uniform distribution to model the probability of false rejection, Kuncheva [4] studied six supervisors: minimum, maximum, average, median, majority vote and Oracle. In practice, the Oracle supervisor does not exist because one does not know in advance the true identity during verification. Therefore, 
it is singled out in this discussion. Among the five classifiers mentioned, Kuncheva found that the average supervisor works the best when the error comes from the two distributions mentioned above.

By considering each sub-component of the multi-source system as an independent classifier, we can use the proof discussed by Bishop [1] (in Chap. 9) to show that the average supervisor satisfies $E_{d} \leq \operatorname{mean}_{j}\left(E_{j}\right)$, instead of $\forall_{j}\left(E_{d} \leq E_{j}\right)$ the "perfect" requirement established earlier. He has shown that a committee of average and weighted average classifiers could perform better than a single classifier. The assumptions here are that each single-modality biometric sub-component is not correlated and that the error of each sub-component has zero mean. The result of proof is shown here:

$$
\begin{aligned}
E_{d} & =\frac{1}{M^{2}} \sum_{j=1}^{M} E_{j} \\
& =\frac{1}{M} \operatorname{mean}_{j}\left(E_{j}\right) .
\end{aligned}
$$

Equation 9 can be rewritten as the following inequality: $E_{d} \leq \frac{1}{\beta} \operatorname{mean}_{j}\left(E_{j}\right)$, which is valid when $1 \leq \beta \leq M$. Equation 9 shows the upper bound of $E_{d}$, which happens when $\beta=M$. The lower bound of $E_{d}$ happens when $\beta=1$. This could be understood as follows: If the errors made by each sub-component are dependent, i.e., they make exactly the same error in the extreme case $\left(\forall_{j 1, j 2}\left(E_{j 1}=\right.\right.$ $\left.E_{j 2}\right)$ ), then $\operatorname{mean}\left(E_{j}\right)=E_{j}=E d$, which implies that $\beta=1$.

Note that the difference between the context in [1] and our context here is that the independency of each sub-component is true and not an assumption. This is because each biometric sub-component operates on different biometric sources.

\subsection{Multi-sample multi-source approach}

It has already been shown that not only averaging scores of multiple sample can reduce noise in the serial process that is made of the sensor-extractor-classifier chain but also that averaging scores of multiple sources can achieve lower error by a factor of the number of biometric sources.

In this section, we wish to combine the two findings above using the strategy that we call multisample multi-source approach (MSMS). In such a system, we assume that there are $M$ biometric modalities and for each modality, $N$ samples are available. Scores made available to this system is denoted as $y_{i, j}$, where $i \in 1, \ldots, N$ and $j \in 1, \ldots, M$. By taking each score $y_{i, j}$ as a channel of the serial process of sensor-extractor-classifier, we can also associate the correspoding error involved, which we denote as $E_{i, j}$.

We argue that Equation 9 used in multi-source biometric system holds as well for multi-sample biometric system, with the weak assumption that the errors $\left(\forall_{i}\left(E_{i, j}\right)\right.$ for a given $\left.j\right)$ are independent and have a zero mean. We can therefore write Equation 9 by changing the index from $j$ to $i$, as follows:

$$
E_{d}=\frac{1}{N} \operatorname{mean}_{i}\left(E_{i}\right)
$$

Violation of such assumption (in our case) results in increase of performance not by a factor of $\mathrm{N}$ but by a factor of $1 \leq \alpha \leq N)$ [1]. To reflect this, we write: $E_{d} \leq \frac{1}{\alpha} \operatorname{mean}_{i}\left(E_{i}\right)$. (Empirical results by the work of Kittler [3] and our result in the later section also support this argument.) We will use the inequality $E_{d} \leq \frac{1}{\alpha} \operatorname{mean}_{i}\left(E_{i}\right)$ to deduce the inequality of the MSSS model:

$$
E_{m s s s_{j}} \leq \frac{1}{\alpha} \operatorname{mean}_{i}\left(E_{i, j}\right)
$$

In the same way, the inequality $E_{d} \leq \frac{1}{\beta} \operatorname{mean}_{j}\left(E_{j}\right)$ can be applied to the SSMS model as follows:

$$
E_{s s m s_{i}} \leq \frac{1}{\beta} \operatorname{mean}_{j}\left(E_{i, j}\right) .
$$


An MSMS model is by its definition a multi-source version of the MSSS model. So, the inequality $E_{d} \leq \frac{1}{\beta} \operatorname{mean}_{j}\left(E_{j}\right)$ that applies to multi-source holds for the MSMS model. Therefore, it is valid to write:

$$
E_{m s m s} \leq \frac{1}{\beta} \operatorname{mean}_{j}\left(E_{m s s s_{j}}\right) .
$$

By replacing inequality 11 into inequality 13 , we can write:

$$
\begin{aligned}
E_{m s m s} & \leq \frac{1}{\beta} \operatorname{mean}_{j}\left(\frac{1}{\alpha} \operatorname{mean}_{i}\left(E_{i, j}\right)\right) \\
E_{m s m s} & \leq \frac{1}{M \beta} \sum_{j}^{M}\left(\frac{1}{N \alpha} \sum_{i}^{N}\left(E_{i, j}\right)\right) \\
E_{m s m s} & \leq \frac{1}{\alpha \beta N M} \sum_{j}^{M} \sum_{i}^{N}\left(E_{i, j}\right) \\
E_{m s m s} & \leq \frac{1}{\alpha \beta N M} \sum_{(i, j)}^{(N, M)}\left(E_{i, j}\right) \\
E_{m s m s} & \leq \frac{1}{\alpha \beta} \operatorname{mean}_{(i, j)}^{(N, M)}\left(E_{i, j}\right) .
\end{aligned}
$$

Therefore, by the MSMS approach, the system error is reduced by a factor within $[1, M N]$ comparing to the average error when each sub-component works inidividually. The combined score via the MSMS approach using the mean operator is similar to the inequality 14. It is defined as follows:

$$
\begin{aligned}
y_{m s m s} & =\operatorname{mean}_{j}\left(\operatorname{mean}_{i}\left(y_{i, j}\right)\right) \\
& =\frac{1}{M} \sum_{j}^{M}\left(\frac{1}{N} \sum_{i}^{N}\left(y_{i, j}\right)\right) \\
& \left.=\frac{1}{N M} \sum_{j}^{M} \sum_{i}^{N}\left(y_{i, j}\right)\right) \\
& =\frac{1}{N M} \sum_{(i, j)}^{(N, M)}\left(y_{i, j}\right) .
\end{aligned}
$$

\section{Database, Experiments and Results}

\subsection{Database}

Briefly, there are 30 persons in the publicly available LSIIT database ${ }^{1}$. It has two types of biometric modalities: face and voice. For each type of biometric modality, 5 out of 10 samples of each person are used for training and the other 5 samples are used for testing. Both the training and test sets are mutually exclusive. For each biometric model, a set of one-versus-all configuration Multi-Layer Perceptrons (MLPs) are used, meaning to say that each MLP is associated with the biometric sample of a single client. In other words, the samples of an associated client is treated as positive examples while all other samples belonging to other clients are treated as negative examples. Each MLP has an output neuron with a sigmoid activation function so that it can estimate the score of the a posteriori probability $p\left(w_{1} \mid \mathbf{x}\right)$ when given a feature $\mathbf{x}$. For a database of 30 persons, 30 MLPs are needed to distinguish face features and the other 30 MLPs are needed to distinguish voice features. More details on feature pre-processing and extraction can be found in [5]. During testing with the previously

\footnotetext{
${ }^{1}$ The LSIIT BAS database is available at http://hydria.u-strasbg.fr/ norman/BAS
} 
unseen 5 samples, the experiment is first carried out with 1 sample, then 2 samples, and so on up to 5 samples.

\subsection{Experiments and Results}

Figure 3 and Figure 4 show the DET curves of face and voice biometric sources on test sets. Table 1 shows the min HTER (minimum point of the half total error rate) in percentage and approximated EER in percentage (a point nearest to FAR $=F R R$ ) of experiment. Note that the threshold is chosen a posteriori on the test data.

Table 1: The HTERs and EERs of face and voice biometric features

\begin{tabular}{|c|c|c|c|c|c|c|}
\hline $\begin{array}{c}\text { No. of } \\
\text { samples } \\
\text { used }\end{array}$ & $\begin{array}{c}\text { Face } \\
\text { min } \\
\text { HTER }\end{array}$ & $\begin{array}{c}\text { Face } \\
\text { EER }\end{array}$ & $\begin{array}{c}\text { Voice } \\
\text { min } \\
\text { HTER }\end{array}$ & $\begin{array}{c}\text { Voice } \\
\text { EER }\end{array}$ & $\begin{array}{c}\text { Combined } \\
\text { min } \\
\text { HTER }\end{array}$ & $\begin{array}{c}\text { Combined } \\
\text { EER }\end{array}$ \\
\hline 1 & 7.184 & 10.000 & 6.897 & 6.897 & 0.805 & 0.805 \\
2 & 2.701 & 3.333 & 4.828 & 6.494 & 0.690 & 0.690 \\
3 & 1.207 & 2.874 & 4.540 & 6.667 & 0.000 & 0.000 \\
4 & 0.000 & 0.000 & 2.126 & 3.333 & 0.000 & 0.000 \\
5 & 0.000 & 0.000 & 2.414 & 3.333 & 0.000 & 0.000 \\
\hline
\end{tabular}

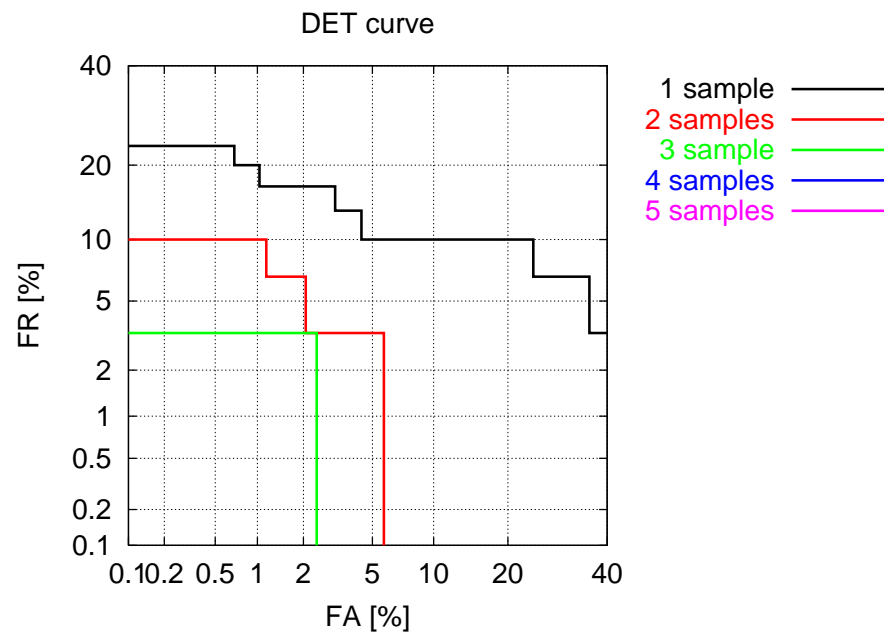

Figure 3: DET curves plotted using 1-5 face samples

The experiment shows that the performance of the MSMS model increases indeed as more and more samples of each biometric source are available. Using the current database, it reaches perfect performance. This is a very encouraging result but in reality, one does not have the luxury of such large pool of data. In our opinion, to further verify this approach, more experiments should be tested with different biometric features and larger databases that have several samples for each biometric source. 


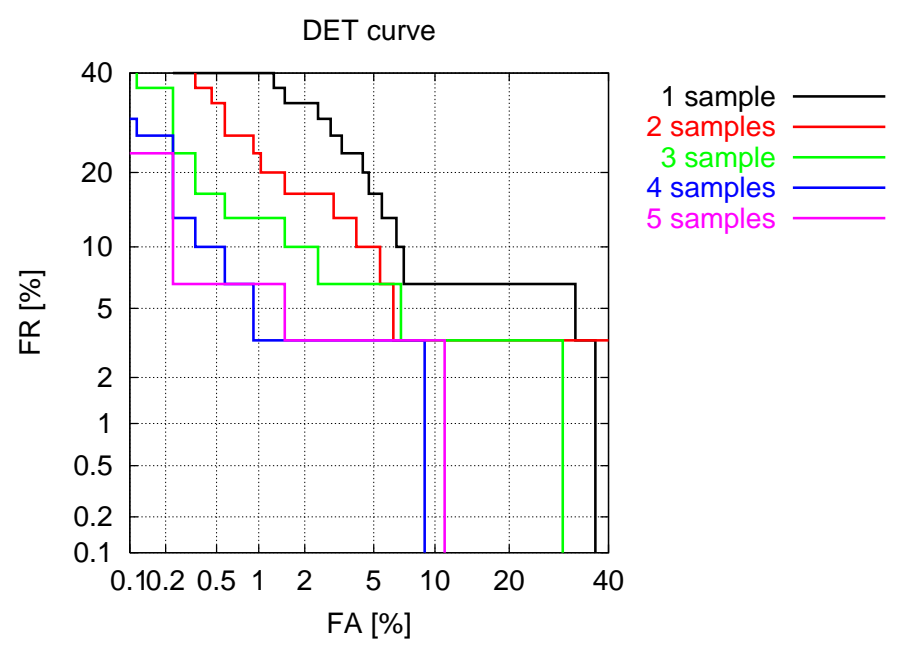

Figure 4: DET curves plotted using 1-5 voice samples

\section{Conclusions}

Biometric authentication can be viewed as a serial process involving a sensor, an extractor, a classifier and a supervisor. One way to increase the reliability of such system is to create several such chain process. Two techniques to do so are using multiple samples and multiple biometric sources. By assuming noise at the score level, it is proven that averaging classifier scores from multiple biometric samples can reduce noise. However, if multiple biometric sources are available, it is proven that the reliability of the joint system can be further increased via averaging. Specifically, by averaging $N$ samples, the joint system will not reach a maximum reduction of error by a factor of $N$ but less due to correlation between samples.

However, by averaging $M$ biometric sources (different biometric modalities), one can achieve a reduction of error approaching a factor of $M$. By combining these two approaches, which we call the multi-sample multi-source approach, one obtains a reduction of error by a factor within $[1, N M]$. This leads to a very significant increase in verification rate.

This hybrid approach is implemented with a set of neural network classifiers and is tested on a face and voice biometric database of 30 persons. Using this small database, a perfect verification is recorded. This result is certainly promising but most importantly, it shows that one can use multiple samples or multiple biometric sources to boost the reliability of the whole system. An interesting application using this approach is in the inconclusive situation, i.e., the final decision score is marginal for acceptance. Under such situation, multi-sample multi-source approach can be taken immediately. This will definitely increase the fault tolerance of intrusion. Furthermore, this approach suggests that it is always beneficial to life-scan longer features (i.e., longer speech signal) and more frames of facial features to increase robustness without adding much cost to the exisiting system.

\section{Acknowledgement}

The main author would like to thank Johnny Mariéthoz for his technical help in using LATEX and several other colleagues, notably Andrew Morris and Guillaume Lathoud over informal discussions on this paper. 


\section{References}

[1] C. M. Bishop, Neural Networks for Pattern Recognition, Oxford University Press, 1999.

[2] L. Hong, A. K. Jain and S. Pankanti, "Can Multibiometrics Improve Performance?" Technical report msu-cse-99-39, MSU-CSE, 121999.

[3] J. Kittler, G. Matas, K. Jonsson and M. Sanchez, "Combining evidence in personal identity verification systems," 1997.

[4] L. I. Kuncheva, "A Theoretical Study on Six Classifier Fusion Strategies," in IEEE Transaction on Pattern Analysis and Machine Intelligence, February 2002, vol. 24, pp. 281-286.

[5] N. Poh and J. Korczak, "Hybrid Biometric Authentication System Using Face and Voice Features," in The 3rd International Conference on AVBPA, 2001, pp. 348-353.

[6] A. Ross, A. Jain and J.-Z. Qian, "Information Fusion in Biometrics," in The 3rd International Conference on Audio-Visual Biometric Person Authentication (AVBPA), 2001, pp. $354-359$.

[7] J. L. Wayman, BIOMETRICS: Person Identification in Networked Society, Kluwer Publishers, chap. 7, 1999. 